\title{
Spontaneous pneumomediastinum due to the Macklin effect: less is more
}

\author{
Guillaume Chassagnon $^{1} \cdot$ Olivier Favelle $^{1} \cdot$ Veronique Derogis $^{2} \cdot{\text { Jean-Philippe } \text { Cottier }^{1}}^{1}$
}

Received: 5 March 2015 / Accepted: 7 March 2015/Published online: 25 March 2015

(C) SIMI 2015

A 17-year-old woman presented to the Emergency Department with the abrupt onset of left hemi-facial swelling. On clinical examination, she had subcutaneous emphysema of the face, neck, and chest. She reported a prior history of asthma, without recent acute exacerbation, and she acknowledged having used cannabis and MDMA (3,4-methylenedioxy- $N$-methylamphetamine) a few hours before. A chest radiograph (Fig. 1a) showed diffuse subcutaneous emphysema associated with a large pneumomediastinum, consistent with the diagnosis of spontaneous pneumomediastinum due to alveolar rupture and the Macklin effect. Although there was no argument in favor of tracheal or esophageal rupture in the anamnesis, a thoracic computed tomography (CT scan) without contrast material injection was finally performed to exclude non-spontaneous pneumomediastinum (Fig. 1b-d). The CT scan confirmed the presence of diffuse pneumomediastinum with subcutaneous emphysema and air dissection into the extrapleural space of the left apex, mimicking a pneumothorax. There was no pneumothorax, rib fracture, or rupture of the trachea or esophagus. Air dissection in the central (peribronchovascular bundles) and peripheral (adjacent to the pleura) pulmonary interstitium, associated with the appearance of alveolar rupture in the middle lobe, confirmed the diagnosis of spontaneous pneumomediastinum due to alveolar rupture and the Macklin effect. The patient was afebrile with normal oxygen saturation, but had

Guillaume Chassagnon gchassagnon@gmail.com

1 Department of Radiology, University Hospital of Tours, 2 Boulevard Tonnelle, 37000 Tours, France

2 Emergency Department, University Hospital of Tours, Tours, France a leukocytosis $(20 \mathrm{~g} / \mathrm{L})$ without elevation of the C-reactive protein (CRP). She was admitted to the thoracic surgery service for observation, and was treated with analgesics. She was discharged after a brief hospitalization of $36 \mathrm{~h}$, and follow-up consultation 3 days later confirmed a marked reduction of the subcutaneous emphysema.

Although spontaneous pneumomediastinum may have a striking clinical presentation, the symptoms are usually self-limited and resolve in a few days. Despite this impressive presentation, physicians should resist the temptation to over-image and hospitalize these patients, as was the case here. The Macklin effect was experimentally demonstrated by Macklin et al. in 1939. It corresponds to an alveolar rupture with air dissection along the interstitial sheaths toward the mediastinum, eventually leading to a pneumomediastinum [1]. Alveolar rupture is responsible for more than $95 \%$ of cases of pneumomediastinum, and is more frequent than esophageal or tracheobronchial disruptions [1]. Identification of the Macklin effect on chest CT scan by visualizing air dissection along perivascular and peribronchial sheaths has been reported in 89-100\% of cases of spontaneous pneumomediastinum, but CT scan confirmation is unnecessary [1,2]. The Macklin effect has also been implicated in $39 \%$ of cases of pneumomediastinum following severe blunt chest trauma [3].

Spontaneous pneumomediastinum occurs predominantly in young males, and is frequently associated with respiratory diseases such as asthma and chronic obstructive pulmonary disease $[2,4]$. A triggering event can be identified in $39-75 \%$ of cases, such as an acute exacerbation of asthma, coughing, inhalation drug abuse, vomiting, physical exertion, the Valsava maneuver, or karaoke singing [2, $4,5]$. Air dissection into the pulmonary interstitium does not affect gas exchange [3]. Fever up to $38.5^{\circ} \mathrm{C}$ and leukocytosis with a CRP level less than $5 \mathrm{mg} / \mathrm{L}$ are 

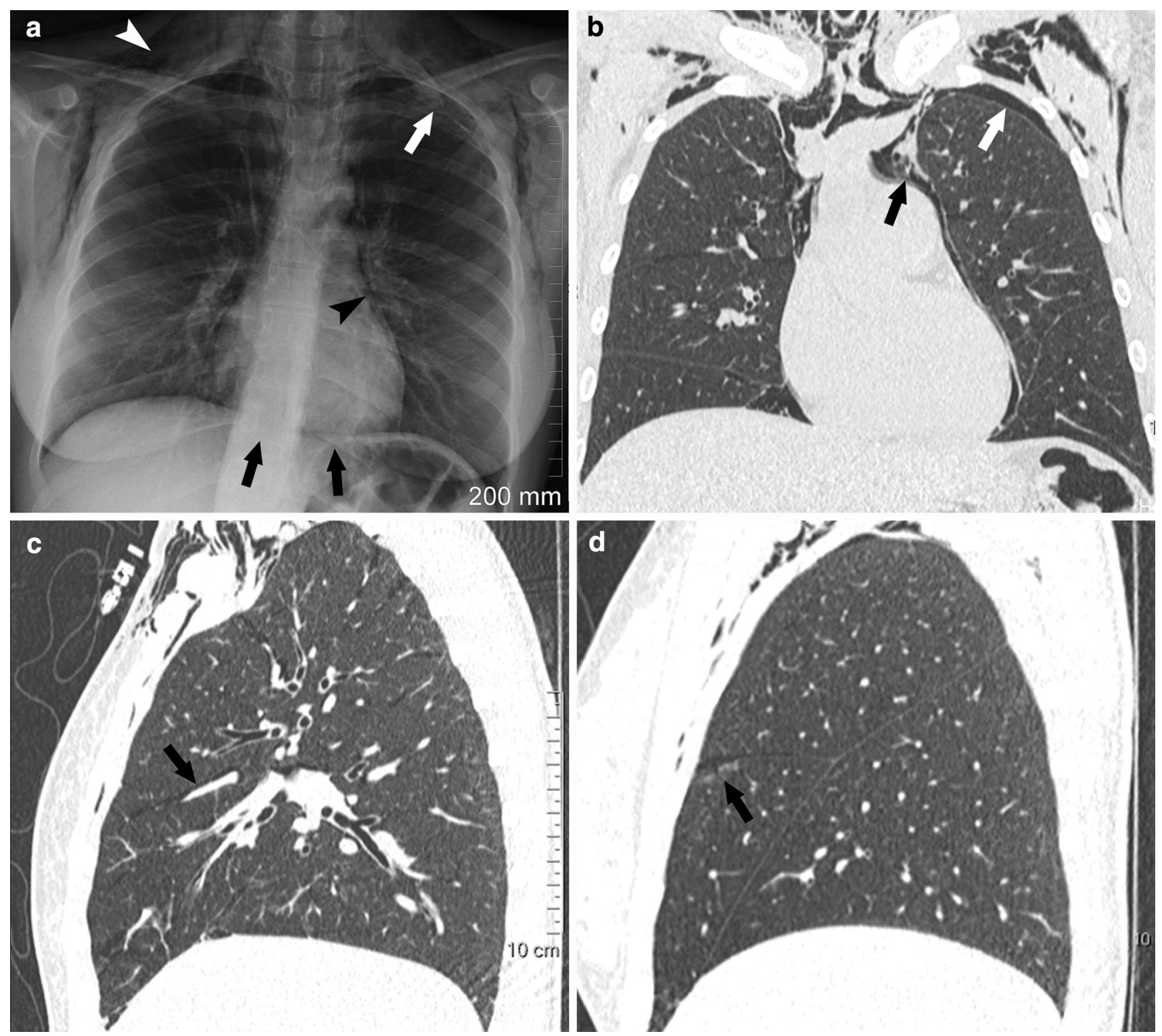

Fig. 1 a Postero-anterior chest radiography demonstrates typical features of pneumomediastinum: subcutaneous emphysema (white arrowhead), extrapleural gas mimicking left pneumothorax (white arrow), a continuous diaphragm (black arrows), and linear density parallel to the left heart border (black arrow head); b coronal reformatted CT scan images confirm the presence of subcutaneous emphysema, pneumomediastinum (black arrow), and air dissection into the extrapleural space, mimicking a left apical pneumothorax

(white arrow); c, d sagittal reformatted CT scan images confirm the Macklin effect by demonstrating air dissection along the peribronchovascular sheaths (arrow in c) and alveolar rupture in middle lobe in contact with air dissection of peripheral pulmonary interstitium (arrow in d). These CT scan images were unnecessary to obtain, but illustrate the mechanism of the Macklin effect leading to a pneumomediastinum when there is an alveolar rupture

frequently encountered in patients with spontaneous pneumomediastinum due to the Macklin effect, and are not associated with an adverse outcome [4]. Prophylactic antibiotics are not necessary, and there is usually no recurrence or complication [2, 4, 5]. To avoid unnecessary hospitalizations, Okada et al. [2] have proposed a fivequestion checklist to select eligible patients for ambulatory care. Thus, patients without (1) fever $>38^{\circ} \mathrm{C}$; (2) oxygen

desaturation $<96 \%$; (3) progressive symptoms; (4) vomiting at the onset; and (5) anxiety should receive ambulatory care. In most of the cases, admission is not necessary and a few hours of high flow oxygen can relieve swelling and chest pain.

Thus, a better understanding of this condition would have avoided unnecessary irradiation of this young woman (the only workup the patient needed was a simple Chest 
X-ray Study). The hospitalization was also not needed since she did not fulfill the criteria that predict an adverse outcome.

Acknowledgments The authors thank Dr. John Scatarige for English language assistance.

\section{Conflict of interest None.}

Statement of human and animal rights All procedures performed in studies involving human participants were in accordance with the ethical standards of the institutional and/or national research committee and with the 1964 Helsinki declaration and its later amendments or comparable ethical standards.

Informed consent No informed consent.

\section{References}

1. Sakai M, Murayama S, Gibo M, Akamine T, Nagata O (2006) Frequent cause of the Macklin effect in spontaneous pneumomediastinum: demonstration by multidetector-row computed tomography. J Comput Assist Tomogr 30:92-94
2. Okada M, Adachi H, Shibuya Y, Ishikawa S, Hamabe Y (2014) Diagnosis and treatment of patients with spontaneous pneumomediastinum. Respir Investig 52:36-40

3. Wintermark M, Schnyder P (2001) The Macklin effect: a frequent etiology for pneumomediastinum in severe blunt chest trauma. Chest 120:543-547

4. Takada K, Matsumoto S, Hiramatsu T, Kojima E, Watanabe H, Sizu M, Okachi S, Ninomiya K (2008) Management of spontaneous pneumomediastinum based on clinical experience of 25 cases. Respir Med 102:1329-1334

5. Campillo-Soto A, Coll-Salinas A, Soria-Aledo V, Blanco-Barrio A, Flores-Pastor B, Candel-Arenas M, Aguayo-Albasini JL (2005) Spontaneous pneumomediastinum: descriptive study of our experience with 36 cases. Arch Bronconeumol 41:528-531 